\title{
Building sustainable global collaborative networks: Recommendations from music studies and the social sciences
}

\author{
*1Patrick E. Savage (Keio University, Japan) \\ *Nori Jacoby (Max Planck Institute for Empirical Aesthetics, Germany) \\ *Elizabeth H. Margulis (Princeton University, USA) \\ Hideo Daikoku (Keio University, Japan) \\ Manuel Anglada-Tort (Max Planck Institute for Empirical Aesthetics, Germany) \\ Salwa El-Sawan Castelo-Branco (Ethnomusicology Institute - Center for Studies in Music and Dance, \\ NOVA University of Lisbon, Portugal) \\ Florence Ewomazino Nweke (University of Lagos, Nigeria) \\ Shinya Fujii (Keio University, Japan) \\ Shantala Hegde (National Institute of Mental Health and Neurosciences, India) \\ Hu Chuan-Peng (Nanjing Normal University, China) \\ Jason Jabbour (UN Environment Program, USA) \\ Casey Lew-Williams (Princeton University, USA) \\ Diana Mangalagiu (University of Oxford, UK and Neoma BS, France) \\ Rita McNamara (Victoria University of Wellington, New Zealand) \\ Daniel Müllensiefen (Goldsmiths, University of London, UK) \\ Patricia Opondo (University of KwaZulu-Natal, South Africa) \\ Aniruddh D. Patel (Tufts University, USA) \\ Huib Schippers (Griffith University, Australia) \\ *These authors contributed equally \\ ${ }^{1}$ Correspondence to: psavage@sfc.keio.ac.jp
}

Please note: We welcome questions, comments, citation, and constructive criticism on this in press manuscript (which may differ from the final published version). Please direct correspondence to psavage@sfc.keio.ac.jp.

Recommended citation: Savage, P. E., Jacoby, N., Margulis, E. H., Daikoku, H., AngladaTort, M., Castelo-Branco, S. E.-S., Nweke, F. E., Fujii, S., Hegde, S., Chuan-Peng, H., Jabbour, J., Lew-Williams, C., Mangalagiu, D., McNamara, R., Müllensiefen, D., Opondo, P., Patel, A., \& Schippers, H. (In press). Building sustainable global collaborative networks: Recommendations from music studies and the social sciences. In E. H. Margulis, D. Loughridge, \& P. Loui (Eds.), The science-music borderlands: Reckoning with the past, imagining the future. MIT Press. Preprint: http://doi.org/10.31234/osf.io/cb4ys

\begin{abstract}
:
Global collaborative networks have been established in multiple fields to move beyond research that over-relies on Western participants and to consider central questions from cross-cultural and epistemological perspectives. As researchers in music and the social sciences with experience building
\end{abstract}


and sustaining such networks, we participated in a virtual symposium on February 7, 2021. to exchange knowledge, ideas, and recommendations, with an emphasis on developing global networks to investigate human music-making. We present $14 \mathrm{key}$ take-home recommendations, particularly regarding 1) enhancing representation of researchers and research participants, 2) minimizing logistical challenges, 3) ensuring meaningful, reproducible comparisons, and 4) incentivizing sustainable collaboration and shared research practices that circumvent research hierarchies. Two overarching conclusions are that sustainable global collaborations should attempt shared research practices including diverse stake-holders, and that we should fundamentally re-evaluate the nature of research credit attribution.

\section{Keywords:}

Diversity, interdisciplinary, shared research practices, research credit attribution

\section{Introduction}

Diversity is one of the key challenges facing society in the 21 st century. In scholarly work, one area this challenge has crystallized around is the over-representation and over-reliance of research on and by societies that are "WEIRD": Western, Educated, Industrialized, Rich, and Democratic (Henrich et al., 2010). While this acronym has been critiqued (Clancy \& Davis, 2019; Barrett, 2020) and even its creators emphasize that "WEIRD" is a rhetorical device not intended to be put in binary opposition with "non-WEIRD" (Apicella et al., 2020; Muthukrishna et al., 2020), the acronym has become popular as a simple way of framing issues of inclusion and representation in academia. In music studies, this problem manifests in areas such as the historical overrepresentation of music by European classical composers and the over-representation of undergraduate students at Western universities in participant samples (Thompson et al., 2019; Jacoby et al., 2020; Savage, In press).

These issues have gained increasing visibility within the mainstream, particularly following calls for decolonial research approaches (Mignolo, 2011) and the rise of the Black Lives Matter movement. Calls to decolonize music studies and make them more inclusive and equitable (e.g., Ewell, 2020; Brown, 2020; Iyer \& Born, 2020; Diamond \& Castelo-Branco, 2021) have recently reached the pages of the New York Times (Powell, 2021) and Fox News (Betz, 2020). In the United States, among other developments, they have triggered important changes in the organizational structure of the Society for Ethnomusicology (SEM) at the highest levels, and have seen the board of the Society for Music Perception and Cognition (SMPC) publish an anti-racism statement (Baker et al., 2020). These changes are part of a broader, long-term international trend, as can be seen in the International Council for Traditional Music (ICTM)'s "Statement and activities in view of decolonization of music and dance studies" (ICTM, 2021), which includes the convention of a year-long series "ICTM Dialogues 2021: Towards decolonization of music and dance studies".

A number of recent global, many-author music science publications highlight both the momentum for change and the challenges that remain. For example: 1) 35 authors published results of rhythm perception experiments involving 923 participants from 39 participant groups in 15 countries (Jacoby et al., Preprint); 2) 19 authors published analyses of 4,709 ethnographic documents and 118 audio recordings of music from around the world (Mehr et al., 2019); 3) 18 authors published a global database of performing arts including analyses of 5,779 songs from 992 societies (Wood et al., Preprint); 4) 20 authors published a critical discussion of challenges and potential for cross-cultural work in music cognition (Jacoby et al., 2020). Some have praised the ambition of these multidisciplinary, global collaborations, but some have also voiced concern about the ways they may actually reinforce pre-existing power structures and hierarchies (e.g., through over-representation of authors 
from well-funded science programs in elite Euro-American universities, and through the use of scientific methods to identify potential cultural "universals"; for discussion, see Russonello, 2017; Rasmussen \& Cowdry, 2018; Savage, 2018; Yong, 2018; Woo, 2019; Jacoby et al., 2020; Loughridge, 2021).

Similar challenges are shared by other fields outside of music studies, which have also grappled with the "WEIRD" concept, its relationship to race and racism (Clancy \& Davis, 2019), and the related issue that "WASP" (Western, Academic, Scientific, Psychology) researchers tend to be over-represented in cross-cultural research (Sinha, 2002; Berry, 2015). Social science fields such as anthropology, economics, and psychology are already making progress on practical solutions for enabling sustainable global collaborative research (e.g., Henrich et al., 2005; Banerjee et al., 2010; Jabbour \& Flaschland, 2017; Purzycki et al., 2017; Moshontz et al., 2018; Broesch et al., 2020; Byers-Heinlein et al., 2020; Urassa et al. 2021; Parker \& Kingori, 2016; Barrett, 2020; Haeleswater et al., 2021).

The aim of this chapter is to provide concrete recommendations for moving beyond the traditional over-reliance on Western music/musicians and toward sustained collaborations that include members of diverse societies throughout the world as equal partners in shared research practices as part of an ecology of knowledges (de Sousa Santos, 2007; Sardo, 2017; Schippers \& Grant, 2016). These recommendations are not intended to be onerous, prescriptive rules but rather a few non-exhaustive ideas to encourage progress and create excitement about future opportunities. Our goal is not to discourage cross-cultural research unless it follows these recommendations, but rather to encourage more such research and provide practical guidance and recommendations to help realize this goal.

Based on the lessons of a previous symposium focused on bridging ethnomusicology and music cognition (Jacoby et al., 2020), the desire to include both a more global representation of participants, and a desire to learn best practices from fields outside of music studies, our three co-first authors (PES, NJ, and EHM) organized a symposium entitled "Building sustainable global collaborative research networks". This symposium featured a group of 23 researchers and practitioners whose expertise was roughly equally distributed between (ethno)musicology, music cognition, and other social sciences (Fig. 1) ${ }^{1}$.

\footnotetext{
${ }^{1}$ The symposium was originally intended to be a 2-day in-person symposium held at the Max PlanckNYU Center for Language, Music, and Emotion (CLaME) in New York City on March 15-16, 2020, but had to be modified due to the COVID-19 pandemic.
} 


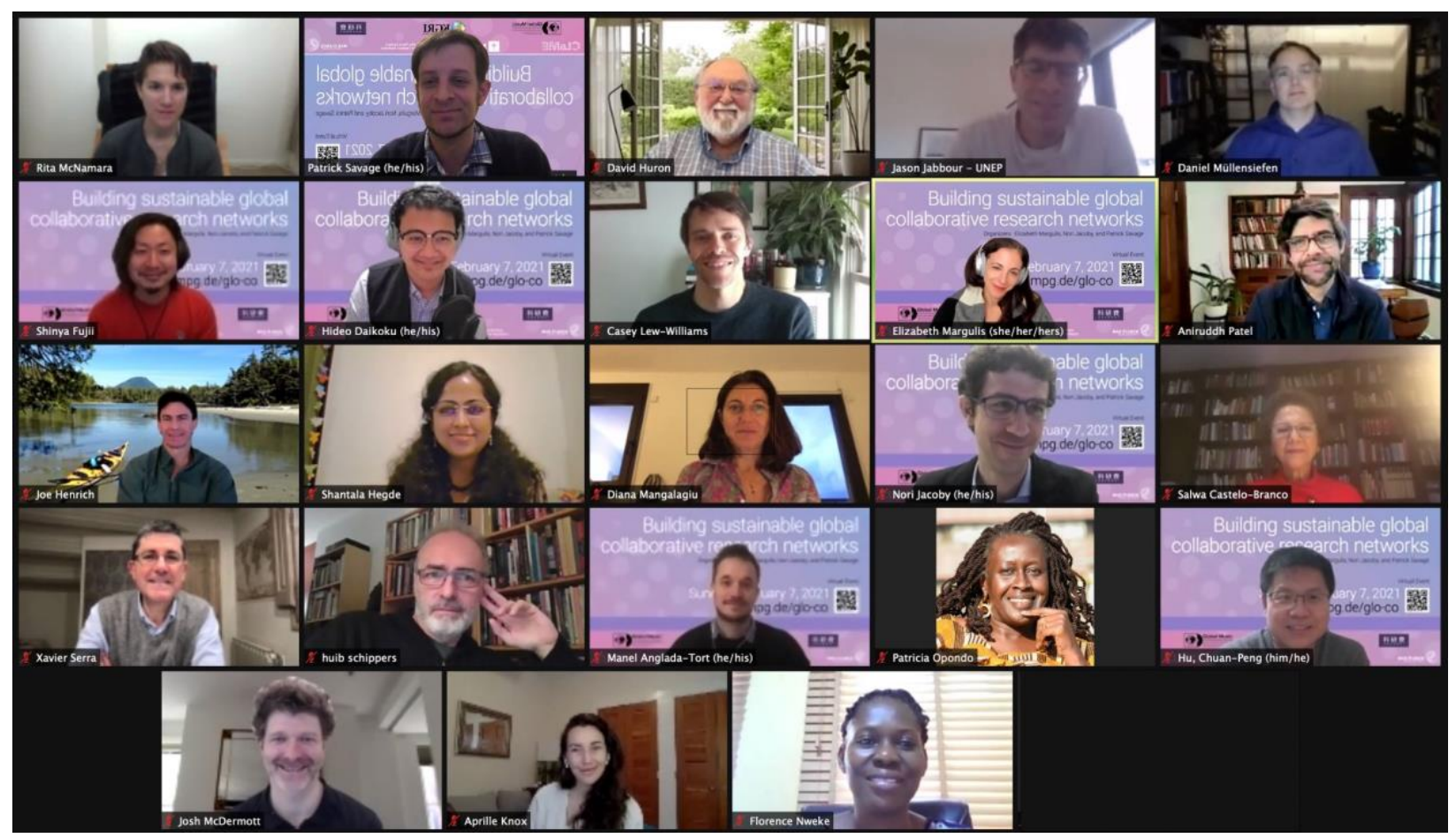

Figure 1. The 23 participants at the Feb 72021 virtual symposium on "Building sustainable global collaborative research networks" (https://www.ae.mpg.de/glo-co).

Prior to the symposium, invitees were asked to submit ideas and resources regarding best practices. After careful review by the symposium organizers four overarching themes emerged: 1) Diversity/inclusion/equity; 2) Logistics; 3) Reproducibility/standardization vs. cultural specificity; and 4) Incentives/attribution/leadership. Participants engaged in discussion about these ideas in small groups of 5-6 people. The following sections synthesize and summarize our shared conclusions about best practices for each of these four key themes.

\section{1) Diversity/inclusion/equity: How do we enhance representation in global collaborations?}

While the importance of diversity is widely recognized, achieving inclusive and equitable representation in global collaborations is easier said than done. Many of the documents cited above by ourselves and others emphasizing diversity in cross-cultural research were nevertheless co-authored mostly or entirely by researchers from elite Euro-American universities (e.g., Broesch et al., 2020; Jacoby et al., 2020). Such imbalances reflect a variety of power structures, including extractive legacies of colonialism as well as practical barriers of language, politics, economics, and disciplinary conventions. Overcoming such legacies and barriers requires rethinking ways of doing research that many of us have come to take for granted. It requires forms of affirmative action to compensate for historical power imbalances and underrepresentation, and requires that we ensure that the goals of the research serve the interests of both the researchers and the communities. Many companies, governments, academic societies, indigenous communities, and other organizations have begun to develop best-practice guidelines for diversity and inclusion, which can vary substantially depending on the specific goals and needs of the organization (e.g., Kirkness \& Barnhardt, 1991; Awesti et al., 2016; Boatright et al., 2018; Chambers et al., 2017; Laland et al., 2020; Nature Editors, 2020; Muru-Lanning, 2020). Below we outline some best practices specifically for building sustainable global collaborative networks. 
Goals/leadership: The goals of the collaboration should be aligned with the needs of the communities that will be involved. The best way to accomplish this kind of goal alignment is to ensure that the voices of the relevant communities are heard and reflected at the highest levels and the earliest stages of a planned collaboration, ideally by involving representatives of these communities in the initial decision-making. The benefits of early co-planning need to be balanced against the realities that: a) involving too many people can reduce the ability to efficiently make high-level decisions, and b) members of under-represented communities are often disproportionately over-burdened with requests to represent that community. Such constraints can result in unintended negative consequences, such as incentivizing the inclusion of so-called "diverse" members in tokenizing ways that "tick the boxes" for diversity on paper only. At minimum, we recommend attempting to identify and recruit stakeholders representing diverse communities at all levels of organization, beginning at the outset of a project and proceeding through shared research practices. Some have created informal lists (e.g., a list of evolutionary human sciences researchers belonging to underrepresented minority groups: https://diversifyehs.mystrikingly.com/) or formal networks (e.g., ICTM's world network of researchers on music and dance from over 100 countries: http://ictmusic.org/world-network) to facilitate such recruitment. One of the topics that are centrally debated in the ICTM at the moment is how to decolonize music and dance research. Lists and networks should also be combined as much as possible with discussions with local stake-holders, and care should be taken to ensure that power imbalances are not perpetuated at local scales (e.g., research on lower-caste musicians performed exclusively with higher-caste local collaborators).

Interdisciplinary collaboration: Global research collaborations are often driven by the interests and funding of scientists, sometimes at the expense of researchers from the humanities or members of the public outside of academia. This is particularly pertinent in cases where the collaboration focuses on research areas, such as music, that involve practices that are frequently unfamiliar to the scientific community, and thus it is essential that researchers sincerely engage with other methods and values. For example, qualitative and quantitative methodologies should be considered, as well as research outputs such as artistic performances, community workshops, blog posts, etc., in addition to academic outputs like peer-reviewed journal articles. For such outputs, it is important to negotiate issues such as authorship and compensation early on. For example, in some societies or disciplines, having one's name listed as a coauthor on a scientific article will have little value compared to being paid as a consultant/research assistant, while in others the reverse may be true. Even within scientific communities, norms regarding authorship and attribution are heterogenous and rapidly evolving. Describing the process taken to involve local co-researchers and advisors can be helpful whether or not they are acknowledged as coauthors (Thompson et al., in prep.).

We recommend attempting to recognize contributions to research networks through financial (e.g., consultation fees, grant co-investigators, experiment costs) and intellectual mechanisms (e.g., coauthorship, author contributions statement, named acknowledgment), as well as ensuring access to, and credit for, research-related outputs (e.g., making archival or field recordings available to community members or providing high-quality video recordings for musicians to use in their own promotional materials). For example, a project measuring global diversity in rhythm perception led by NJ (Jacoby et al., Preprint) includes 34 authors - including scientists, (ethno)musicologists, and musicians - from 15 countries (Germany, Austria, Sweden, USA, UK, Canada, Japan, South Korea, China, Chile, Bolivia, Uruguay, Mali, India, Turkey) and names 51 individuals and organizations in the acknowledgments. 
It is imperative to ensure that the recognition researchers and participants receive is specifically of value to them. This may not always necessarily mean formal coauthorship (e.g., Araújo \& Cambria, 2013; Miguel, 2018). For example, in a project exploring musical diversity in India (Daikoku et al., 2021) the graduate student leading the project (HD) is from India and receives both financial support (stipend and tuition funded by Yamaha) and intellectual credit (first authorship). He is working with musicians in India to take music lessons and conduct interviews and experiments, and these musicians receive financial compensation but not coauthorship. It is also important to recognize that in some communities it may be considered inappropriate to explicitly discuss such reward mechanisms - as always these suggestions should be adapted as much as possible to the norms of the local context.

Language/geography/accessibility: The current concentration of academic power in English-speaking countries incentivizes us to organize events and collaborations in English in these countries - just like the one that led to this chapter (which is also written in English). This marginalizes members of nonAnglophone communities and creates barriers to their inclusion in global research networks. Providing travel funding can minimize some of the economic barriers, but does not solve other problems such as language barriers, visa restrictions, and other factors that can limit participation. While it may seem inefficient to hold meetings in other countries using languages other than English given the additional costs for travel, translation, etc., such short-term costs are necessary in order to build long-term sustainability. ICTM is an example of an academic society that has successfully organized in-person world conferences in diverse countries with multilingual translation (e.g., South Africa in 2009, Kazakhstan in 2015, China in 2018, Thailand in 2019) and virtual events (e.g., the "Decolonization Dialogues" mentioned above [ICTM 2021]). The rapid normalization of virtual participation due to the COVID-19 pandemic may help to reduce barriers and costs associated with travel, but will not solve issues of language and may create other imbalances. To actively reduce such barriers to participation, we recommend to organize events in diverse geographic locations using diverse languages, and provide opportunities for translation and virtual participation that are as accessible as possible (e.g., for participants with disabilities, caregiver obligations, etc.). This may go beyond literal translation of language to include conceptual translation of concepts, which may need to be entirely rethought and reformulated in terms more relevant to different communities.

\section{2) Logistics: How can we minimize logistical challenges in global collaborations?}

Even research within a single society involves substantial logistical challenges, and these are amplified drastically when conducting global collaborative research. Different societies have different rules, norms, and institutional structures. Collaborating across these in a meaningful way requires careful planning, including aspects such as organizational structure, funding, and ethical review.

Project management: Building and sustaining a global collaborative network requires a clear leadership structure that balances flexibility and agency for individual researchers/labs in different societies with a clear unifying vision and strategy. In service of this aim, we recommend balancing top-down (e.g., standardized protocols) and bottom-up (e.g., delegating local adaptations) project management approaches. For example, the Evolution of Morality Project developed standardized and validated methods for measuring cooperation and morality cross-culturally, but adapted these according to the relevant local 
belief systems of each of the eight societies investigated (Purzycki et al., 2017). Likewise, Jacoby et al. (Prerint) provided researchers in different societies with thoroughly piloted and well-documented standardized equipment and protocols for the rhythm experiments, but partially delegated decisions about translation and appropriate participant sampling to local researchers (while maintaining consultation to ensure sampling rationale remained consistent across societies). The equipment was designed to be portable and flexible, allowing researchers to conduct experiments even in remote areas with unreliable infrastructure.

Funding: Funding logistics can be particularly complicated for global collaborations. Economic and geopolitical power imbalances mean that some countries offer more funding than others, and may limit the countries to which funds can be transferred. The complexity of global collaborations often requires retracing steps and pivoting to different approaches at key junctures in research, making it challenging to specify and follow long-term funding timelines. They also may require extra funding needs that may be difficult to fully anticipate at the time of funding applications. For example, the ManyBabies Consortium (Byers-Heinlein et al., 2020), in an ongoing collaboration with scientists from various nations in Africa, did not originally budget for IRB fees, which are not usually charged in Western universities but are common in some academic communities. After obtaining initial funding, they learned that many collaborators would need to pay USD $\$ 500$ to their institutions. This unanticipated expense impacted other components of the project budget. We recommend identifying collaborators prior to writing grants, and then jointly crafting detailed budgets that accommodate the range of expenses involved in global collaborations.

Ethical review: Many of the logistical issues involved in global collaborations intersect with ethical issues involving disparities across different sites. These range from more specific practical issues (e.g., the compensation, data management, and anonymity of participants) to more general ones (e.g., how can we ensure the research is helping and not hurting the local community?). Institutional review boards (IRBs) are a formal mechanism for evaluating such issues. IRBs have been criticized as "more concerned with protecting the institution than research participants" (Grady, 2010), but if well stewarded, they may be used to clarify rights and obligations for everyone early in the project, avoiding unfortunate situations later. For example, $\sim 1,000$ of the $\sim 6,000$ music recordings from indigenous groups in North America and Australia will not be available for listening at the Global Jukebox (Wood et al., Preprint) until time-consuming negotiations with representatives of each individual group are completed. These problems might have been mitigated if such issues had been clarified in IRB protocols at the beginning of the project (though note that the Global Jukebox project began over a half-century ago before IRB review had become standard research practice). For societies without local IRBs, we recommend applying the highest standards to protect the rights of participants and avoid ethics dumping (Schroder et al., 2018). It is also crucial to realize that the very principles of IRB may not operate well in many cultures, for instance those with a hierarchy or consensus culture that makes individual consent meaningless. As in the "project management" section above, we recommend adopting a combined top-down/bottom-up approach, in which general standardized IRB protocols are prepared in consultation with diverse team members, and these standardized protocols are then adapted to local institutions as necessary.

Accessibility: Setting up easily accessible online data collection can help reduce logistical costs of traveling to remote areas, especially during times when travel is not physically possible (e.g., the current COVID-19 pandemic). Some communities benefit from the ability to use mobile phones, where experiments, 
questionnaires, etc. can be implemented using responsive web-based apps. These methods are sometimes insufficiently embedded in the cultural context, and don't allow much control over the conditions within which the experiment is taking place. However, such concerns can be mitigated by taking appropriate precautions (e.g., pre-screening tasks, data quality checks, bonus payments, etc). This is true even for highly controlled experiments, such as infant research (Tran et al., 2017), language production tasks (Vogt et al., 2021), or iterated tapping experiments (Jacoby et al., preprint). Online data collection has demonstrated considerable success (Kohavi \& Thomke, 2017) and is likely to be increasingly a mainstay of research methodology. We recommend including online options when feasible to enhance accessibility and diversity.

\section{3) How can we ensure meaningful, reproducible comparisons on a global scale?}

Reproducibility: Increasingly popular open science practices enhance transparency and reproducibility through free sharing of data, analysis code, stimuli/protocols, pre-registered hypotheses, and research reports via standardized repositories such as the Open Science Framework, Github, Zenodo, arXiv, and related preprint servers.However, the need to preserve and promote diversity sometimes works against this tendency toward openness and standardization. Many historically under-represented minorities are wary of having their personal data documented and shared in forms they cannot control after experiencing atrocities and humiliation in the name of science (Brandt, 1978; van Noorden, 2020). Here again, we recommend using the IRB review process as an opportunity to specify data-sharing procedures and grapple with the accompanying ethical considerations early on. In addition, we recommend prioritizing sharing stimuli/protocols and analysis code even in cases where sharing participants' data is more complex.

Standardization/translation: Even when diverse participants provide informed consent, cross-cultural differences mean that standardized research metrics like IQ can at best be considered meaningful only when interpreted cautiously, and at worst can actually be meaningless or actively harmful (European Human Behaviour and Evolution Association, 2020). The same caveat likely applies to other standardized attempts to measure things like "musicality", "musical sophistication" (Müllensiefen et al., 2014), or "musical IQ" (Neely, 2020) that lack cross-culturally universal definitions (Savage, 2019). Building sustainable global collaborations requires us to constructively address such challenges. We recommend collaborating with local researchers to develop/translate/adapt questionnaires and experimental stimuli/protocols to ensure that the resulting data can be used to make meaningful comparisons. The limitations of existing inventories should be acknowledged, and possibilities for completely reframing ideas from alternative perspectives rather than simply translating them should be explored (cf. Harris, 1976). When possible, subjective self-report measures (e.g., self-reported daily practice time) should be combined with objective measures (e.g., beat synchronization; Müllensiefen et al., 2014). While no index for terms such as "musicality" and "musicians" will ever be perfect, we believe that working toward creating indices that are more thoroughly cross-culturally validated than existing ones would be a constructive goal. Promising steps have already been made through cross-cultural collaborations (e.g., a Chinese translation of the Gold-MSI musicality index; Lin et al., 2019). For more discussion and critical analyses of these concepts, see chapters in this volume by Iyer, Patel, Mundy, and Ilari \& Habibi;.

One possibility to consider is to aim for comparability at the conceptual level of the same latent construct that is to be measured in different cultures. For example, for the construct "musical expertise", researchers from different musical cultures might agree that measurement on a uni-dimensional scale ranging from low 
to high would be meaningful in their own culture. Once this is agreed upon, it might be possible to create different inventories with question items specific to each culture that measure the same construct by asking different questions. Similarly, researchers might agree that a specific musical skill is important in their cultures (e.g. intonation accuracy). Then, several different versions of a perceptual test could be developed, each version using stimuli that are culturally meaningful within each culture and each version being validated with a sample of participants from the corresponding musical culture. Scores on the task can then still be made comparable if a scoring metric is used that does not depend on the items of the individual test (e.g., item response scoring or Rasch modelling). Such efforts should take advantage of existing methods for establishing comparability of scale or questionnaire-based measures across groups using techniques like measurement invariance (e.g., Fischer, 2004; Chen, 2008; Fischer \& Poortinga, 2018; Boer et al., 2018; Jeong \& Lee, 2019).

Sampling: A core scientific principle is that the population sampled for a given study should be representative of the population to which the conclusions are to be generalized. But given the extreme cultural diversity within and between populations, what does it even mean to say one group of humans is "representative" of another? The "WEIRD" problem described above is increasingly recognized as a major sampling limitation, but simply sampling from "non-WEIRD" societies does not solve the problem, and may in fact exacerbate it (if, for example, the groups are over-essentialized in misleading ways). Such overly simplistic approaches also risk failing to acknowledge the massive diversity within societies, and failing to capture the full range of human cultural diversity (Barrett, 2020). There are often also major, theoretically-relevant differences within a given country or society, such as age, gender, race, or musicianship (Taras et al., 2009). Controlling for all such variables in cross-cultural research is often impossible, but we recommend documenting and justifying sampling and inclusion criteria to help increase the generalizability and reproducibility of a given study, and to prevent overinterpretation. For example, because Jacoby et al. (Preprint) were interested in cross-cultural diversity in rhythm perception, they chose to recruit participants with extensive training in local non-Western musical traditions as well as two types of control participants with similar demographics who had either 1) training in Western musical traditions, or 2) no formal musical training. Ultimately, it was impossible to control for all demographic factors including education and age, but acknowledging such limitations is also an important part of sustainability. Concepts such as "cultural distance" (Muthukrishna et al., 2020) may be useful to help control for cross-cultural similarities and differences (such approaches can in principle simultaneously address diversity within and between societies; cf. Rzesuztek et al., 2012 for a musical example).

\section{4) How can we design systems that will promote sustainable global collaborations?}

Many of the barriers to sustainable global collaborations stem from the systemic nature of research incentive systems such as publication, funding, and hiring practices. While such global systems cannot be easily changed, a number of strategies may help researchers to work effectively within them while gradually changing them to increase equity and sustainability.

Leadership/credit: Historical research assessment systems emphasizing first-author or sole-authored academic publications disincentivize truly interdisciplinary and truly global collaborations, which require sustained investment from multiple individuals from multiple disciplines. It can be hard to interest researchers in investing in collaborations if their name will end up in the middle of the author list where 
evaluation committees do not value it. Within the current system, effective strategies for incentivizing collaboration include things like negotiating financial, intellectual, and data-sharing mechanisms to allow coauthors to receive credit for sub-aspects of the project. For example, local researchers can be provided priority data access or first/co-first authorship on related papers (e.g., journal special issues, edited volumes; cf. Henrich et al., 2004; Apicella et al., 2020) based on the same data. Financial incentives can also help, such as providing consulting fees upon completion of data collection. Ultimately, however, solving these problems will require a fundamental reevaluation of the nature of research credit attribution (cf. Kaiser, 2018; Holcombe, 2019).

Multidisciplinarity: Effective global collaborations require researchers to work across disciplines within academia and to work with local communities, government funders, non-governmental organizations, private industry, and other non-academic stakeholders. Communicating across disciplines and across diverse stakeholders is challenging and can take extra time to ensure everyone feels included, valued, and believes that the collaboration is in their own interest. Nevertheless, we recommend developing shared research practices (Sardo, 2017) that include and synthesize the diverse value systems of community stakeholders to maximize long-term sustainability. For example, the 2018 workshop that preceded the current symposium involved multiple days of long and sometimes very heated discussion between ethnomusicologists and music cognition researchers (Jacoby et al., 2020). Ultimately, though, it led to increased interdisciplinary goodwill and collaborative spirit, as well as the realization that important voices were missing from the discussion, an omission that the organization of the current symposium attempted to address. At the current symposium we discussed the lack of voices of musicians/performers and the need to accommodate the different goals and incentives that might differ between performers and academics, and such iterated dialogues will be necessary to facilitate sustainable long-term collaborations.

Intergenerational sustainability: Building sustainable global collaborations is a long-term goal that will require long-term strategies to achieve. By adopting the recommendations listed above, we can build infrastructures and systems to make global collaborations easier over time, as existing networks grow and stimulate additional funding and opportunities for members of historically underrepresented communities to become involved. To ensure such long-term intergenerational sustainability, we recommend that more senior members actively recruit and incentivize more junior members from diverse backgrounds. This can include strategies like recruiting and securing funding for graduate students and postdocs from developing countries, co-authoring grant applications led by researchers at institutions in these countries, and creating incentives to encourage and reward the next generation of researchers for investing in global collaborations.

\section{Conclusion}

Box 1 condenses and summarizes the 14 key take-home recommendations summarized in this chapter. These recommendations are ambitious, and we have all failed to achieve them in the past. For example, having three white researchers organizing the symposium that led to this chapter using only English violated recommendation 1.1 to involve diverse stakeholders at all levels of organization and recommendation 1.3 to use diverse languages. But the perfect should not be the enemy of the good. We also believe it's important to learn from past failures and to set goals that we may not always be able to meet, but should nevertheless aim to strive for. As stated in the introduction, these recommendations are not intended to be onerous, 
prescriptive rules but rather a few non-exhaustive ideas to encourage progress and create excitement about future opportunities.

1. Diversity: How do we enhance representation in global collaborations?

1. Identify and recruit stakeholders representing diverse communities at all levels of organization and all stages of a project.

2. Recognize contributions to research networks through financial (e.g., consultation fees) and intellectual (e.g., coauthorship) mechanisms. Ensure access to, and credit for, researchrelated outputs (e.g., audiovisual recordings).

3. Organize events in diverse geographic locations using diverse languages, providing accessible options for translation and virtual participation

2. Logistics: How can we minimize logistical challenges in global collaborations?

1. Balance top-down (e.g., standardized protocols) and bottom-up (e.g., delegating local adaptations) project management approaches.

2. Work with a diverse team to draft detailed but flexible budgets that can accommodate the expenses involved in global collaborations.

3. Prepare standardized IRB protocols in consultation with diverse team members, and adapt these standardized protocols to local institutions as necessary.

4. Include online options for data collection when feasible to enhance accessibility.

3. Comparison: How can we ensure meaningful, reproducible comparisons on a global scale?

1. Use the IRB review process as a chance to specify data sharing procedures and accompanying ethical considerations early on.

2. Collaborate with local researchers to develop/translate/adapt/reframe questionnaires and experimental stimuli/protocols.

3. Document and justify sampling and inclusion criteria.

4. Incentives: How can we design systems that will promote sustainable global collaborations?

1. Negotiate financial, intellectual, and data-sharing mechanisms to allow coauthors to receive appropriate credit.

2. Develop shared research practices that include and synthesize the value systems of diverse stakeholders.

3. More senior members should actively recruit and incentivize more junior members from diverse backgrounds.

4. Fundamentally reevaluate the nature of research credit attribution.

Box 1: List of 14 key take-home recommendations.

The current symposium participants do not represent any formal consortium or plan any joint projects with this group as a whole. Rather, they were invited and accepted due to their shared interests and experiences in crosscultural research and their unique perspectives, while attempting to balance representation across multiple dimensions including gender, ethnicity, geography, seniority, and discipline. We are not attempting to establish an exclusive "power clique", but rather to invite and encourage anyone who is interested and shares similar ideas to do cross-cultural research.

We recognize that our list of recommendations reflects our priorities and experiences that have been shaped by our backgrounds as researchers in music studies and the social sciences. These may not necessarily reflect the full range of recommendations and priorities that we may have come up with had we included an even more diverse range of stake-holders (e.g., representatives from community interest groups, 
professional artists, corporations, government). We hope to include and learn from such perspectives and voices in future steps.

We hope that by the time the next generation is organizing similar symposia, the recommendations in Box 1 will seem to be so obvious as hardly worth stating, and look forward to seeing future developments toward equitable and sustainable global research collaborations.

\section{Author contributions:}

PES, JN, and EHM conceived, planned, and organized the symposium, and PES, JN, EHM, and SF obtained funding to hold it. All authors contributed recommendations and ideas prior to and during the symposium, and took part in discussion and editing of the resulting manuscript. EHM drafted the initial manuscript outline, PES wrote the first full draft, and NJ and EHM edited this draft before circulating to other authors. HD and MA-T assisted with symposium preparation and logistic support. Other authors are listed in alphabetical order.

\section{Acknowledgments:}

We thank Dana Bevilacqua for extensive initial planning for our symposium entitled "Building sustainable global collaborative research networks" (https://www.ae.mpg.de/glo-co), which was originally scheduled for March 15th, 2020 at the Max Planck-NYU Center for Language, Music, and Emotion (CLaME) in New York City, and eventually held virtually on Feb 7th, 2021 due to COVID-19. We thank Joseph Henrich, David Huron, Aprille Knox, Josh McDermott, and Xavier Serra for their participation at the symposium and Dorsa Amir, who contributed initial ideas but was unable to attend the symposium after it was postponed. We thank Bill Thompson, Alyssa Crittenden, Gabe Zuckerberg, Jim Sykes, Psyche Loui, Deirdre Loughridge, and participants at the "Science Music Borderlands" Zoom workshop on May 21, 2021 for feedback on a previous draft of this chapter and discussion of other chapters in this edited volume.

The "Building sustainable global collaborative research networks" symposium was funded by a Japan Society for the Promotion of Science Grant-in-aid (Grant No. 19KK0064) and a Keio Global Research Institute Startup Grant. The "Science Music Borderlands" workshop was funded by Princeton University. HD is supported by funding from the Yamaha corporation.

\section{References:}

Apicella, C., Norenzayan, A., \& Henrich, J. (2020). Beyond WEIRD: A review of the last decade and a look ahead to the global laboratory of the future. Evolution and Human Behavior, 41(5), 319329. https://doi.org/10.1016/j.evolhumbehav.2020.07.015

Araújo, S., \& Cambria, V. (2013). Sound praxis , poverty, and social participation: Perspectives from a collaborative study in Rio de Janeiro. Yearbook for Traditional Music, 45, 28-42.

Awesti, A., Flinders, M., \& Savigny, H. (2016) Pursuing the diversity and inclusion agenda: the PSA in the UK. European Political Science, 15 (4), 508-518. 
Baker, D. J., Belfi, A., Creel, S., Grahn, J., Hannon, E., Loui, P., Margulis, E. H., Schachner, A., Schutz, M., Shanahan, D., \& Vuvan, D. T. (2020). Embracing anti-racist practices in the music perception and cognition community. Music Perception, 38(2), 103-105. https://doi.org/10.1525/mp.2020.38.2.103

Banerjee, A., Duflo, E., Goldberg, N., Karlan, D., Osei, R., Parienté, W., Shapiro, J., Thuysbaert, B., \& Udry, C. (2015). A multifaceted program causes lasting progress for the very poor: Evidence from six countries. Science, 348(6236). https://doi.org/10.1126/science.1260799

Barrett, H. C. (2020). Towards a cognitive science of the human: Cross-cultural approaches and their urgency. Trends in Cognitive Sciences, 24(8), 620-638. https://doi.org/10.1016/j.tics.2020.05.007

Berry, J. W. (2015). Global psychology: implications for cross-cultural research and management. Cross Cultural Management: An International Journal, 22(3), 342-355. https://doi.org/10.1108/ccm-03-2015-0031

Betz, B. (2020, August 4). Music faculty at Texas college stokes ire for suggesting music theory isn’t white supremacist. Fox News. https://www.foxnews.com/us/music-faculty-texas-college-musictheory-white-supremacist

Boatright, D., Branzetti, J., Duong, D., Hicks, M., Moll, J., Perry, M., Pierce, A., Samuels, E., Smith, T., Angerhofer, C. and Heron, S. (2018). Racial and ethnic diversity in academic emergency medicine: how far have we come? Next steps for the future. AEM Education and Training, 2, S31-S39.

Boer, D., Hanke, K., \& He, J. (2018). On detecting systematic measurement error in cross-cultural research: A review and critical reflection on equivalence and invariance tests. Journal of CrossCultural Psychology, 49(5), 713-734. https://doi.org/10.1177/0022022117749042

Brandt, A. M. (1978). Racism and research: The case of the Tuskegee Syphilis Study. The Hastings Center Report, 8(6), 21-29. https://doi.org/10.2307/3561468

Broesch, T., Crittenden, A., Beheim, B., Blackwell, A., Bunce, J., Colleran, H., Hagel, K., Kline, M. A., McElreath, R., Nelson, R., Pisor, A., Prall, S., Pretelli, I., Purzycki, B. G., Quinn, E., Ross, C., Scelza, B., Starkweather, K., Stieglitz, J., \& Borgerhoff Mulder, M. (2020). Navigating crosscultural research: Methodological and ethical considerations. Proceedings of the Royal Society B Biological Sciences, 287(20201245). https://doi.org/10.1098/rspb.2020.1245

Brown, D. (2020, June 12). An open letter on racism in music studies: Especially ethnomusicology and music education. My People Tell Stories. https://www.mypeopletellstories.com/blog/openletter

Byers-Heinlein, K., Bergmann, C., Davies, C., Frank, M. C., Hamlin, J. K., Kline, M., Kominsky, J. F., Kosie, J. E., Lew-Williams, C., Liu, L., Mastroberardino, M., Singh, L., Waddell, C. P. G., Zettersten, M., \& Soderstrom, M. (2020). Building a collaborative psychological science: Lessons learned from 
ManyBabies 1. Canadian Psychology/Psychologie canadienne, 61(4), 349-363. https://doi.org/10.1037/cap0000216

Chambers, D., Preston, L., Topakas, A., de Saille, S., Salway, S., Booth, A., Dawson, J. and Wilsdon, J. (2017). Review of diversity and inclusion literature and an evaluation of methodologies and metrics relating to health research. https://wellcome.org/sites/default/files/review-of-diversityand-inclusion-literature.pdf

Chen, F. F. (2008). What happens if we compare chopsticks with forks? The impact of making inappropriate comparisons in cross-cultural research. Journal of Personality and Social Psychology, 95(5), 1005-1018. https://doi.org/0.1037/a0013193

Clancy, K. B. H., \& Davis, J. L. (2019). Soylent is people, and WEIRD is white: Biological anthropology, whiteness, and the limits of the WEIRD. Annual Review of Anthropology, 48, 169-186. https://doi.org/10.1146/annurev-Anthro-102218-011133

Daikoku, H., Wood, A. L. C., \& Savage, P. E. (2021). Musical diversity in India: A preliminary computational study using Cantometrics. Keio SFC Journal, 20(2), 34-61.

Diamond, B., \& Castelo-Branco, S. E.-S. (Eds.). (2021). Transforming ethnomusicology Volume II: Political, social \& ecological Issues. Oxford University Press.

Ewell, P. A. (2020). Music theory and the white racial frame. Music Theory Online, 26(2). https://doi.org/10.30535/mto.26.2.4

Fischer, R. (2004). Standardization to account for cross-cultural response bias: A classification of score adjustment procedures and review of research in JCCP. Journal of Cross-Cultural Psychology, 35(3), 263-282. https://doi.org/10.1177/0022022104264122

Fischer, R., \& Poortinga, Y. H. (2018). Addressing methodological challenges in culture-comparative research. Journal of Cross-Cultural Psychology, 49(5), 691-712. https://doi.org/10.1177/0022022117738086

Grady, C. (2010). Do IRBs protect human research participants? JAMA - Journal of the American Medical Association, 304(10), 1122-1123. https://doi.org/10.1001/jama.2010.1304

Haelewaters, D., Hofmann, T. A., \& Romero-Olivares, A. L. (2021). Ten simple rules for Global North researchers to stop perpetuating helicopter research in the Global South. PLoS Computational Biology, 17(8), e1009277. https://doi.org/10.1371/journal.pcbi.1009277

Harris, M. (1976). History and significance of the emic/etic distinction. Annual Review of Anthropology, 5(1), 329-350.

Henrich, J., Boyd, R., Bowles, S., Camerer, C., Fehr, E., \& Gintis, H. (Eds.). (2004). Foundations of human sociality: Economic experiments and ethnographic evidence from fifteen small-scale societies. Oxford University Press. 
Henrich, J., Boyd, R., Bowles, S., Camerer, C., Fehr, E., Gintis, H., McElreath, R., Alvard, M., Barr, A., Ensminger, J., Henrich, N. S., Hill, K., Gil-White, F., Gurven, M., Marlowe, F. W., Patton, J. Q., \& Tracer, D. (2005). "Economic man" in cross-cultural perspective: Behavioral experiments in 15 small-scale societies. Behavioral and Brain Sciences, 28, 795-855. https://doi.org/10.1017/S0140525X05000142

Holcombe, A. (2019). Farewell authors, hello contributors. Nature, 571, 147. https://doi.org/10.1038/d41586-019-02084-8

International Council for Traditional Music. (2021). ICTM statement and activities in view of decolonization of music and dance studies. http://ictmusic.org/documents/decolonization$\underline{\text { music-dance-studies }}$

Iyer, V., \& Born, G. (2020, February 18). Of musicalities and musical experience: Vijay Iyer and Georgina Born in conversation. Wigmore Hall Podcasts. https://wigmorehall.org.uk/podcasts/of-musicalities-and-musical-experience-vijay-iyer-and-georgina-born-inconversation

Jabbour, J., \& Flachsland, C. (2017). 40 years of global environmental assessments: A retrospective analysis. Environmental Science and Policy, 77, 193-202. https://doi.org/10.1016/j.envsci.2017.05.001

Jacoby, N., Margulis, E., Clayton, M., Hannon, E., Honing, H., Iversen, J., Klein, T. R., London, J., Mehr, S., Pearson, L., Peretz, I., Perlman, M., Polak, R., Ravignani, A., Savage, P. E., Steingo, G., Stevens, C., Trainor, L., Trehub, S., ... Wald-Fuhrmann, M. (2020). Cross-cultural work in music cognition: Methodologies, pitfalls, and practices. Music Perception, 37(3), 185-195. https://doi.org/10.1525/mp.2020.37.3.185

Jacoby, N., Polak, R., Grahn, J. A., Cameron, D. J., Lee, K. M., Godoy, R., Undurraga, E. A., Huanca, T., Thalwitzer, T., Doumbia, N., Goldberg, D., Margulis, E., Wong, P. C. M., Jure, L., Rocamora, M., Fujii, S., Savage, P. E., Ajimi, J., Konno, R., ... McDermott, J. H. (Preprint). Universality and cross-cultural variation in mental representations of music revealed by global comparison of rhythm priors. PsyArXiv preprint. https://doi.org/10.31234/osf.io/b879v

Jeong, S., \& Lee, Y. (2019). Consequences of not conducting measurement invariance tests in crosscultural studies: A review of current research practices and recommendations. Advances in Developing Human Resources, 21(4), 466-483. https://doi.org/10.1177/1523422319870726

Kirkness, V. J., \& Barnhardt, R. (1991). First Nations and higher education: The four R's - respect, relevance, reciprocity, responsibility. Journal of American Indian Education, 30(3), 1-15.

Kiser, G. L. (2018). No more first authors, no more last authors. Nature, 561(7724), 435. https://doi.org/10.1038/d41586-018-06779-2

Kohavi, R., \& Thomke, S. (2017). The surprising power of online experiments. Harvard Business Review, 95(5), 74-82. 
Laland, K., Kendal, R., Jordan, F., \& Creanza, N. (2020). Cultural Evolution Society: Guidelines for $\begin{array}{lllll}\text { organising } & a & \text { diverse } & \text { conference workshop. }\end{array}$ https://culturalevolutionsociety.org/files/ces_conferenceworkshop_guidelines_3_nov202020201112075628.pdf

Lin, H.-R., Kopiez, R., Müllensiefen, D., \& Wolf, A. (2019). The Chinese version of the Gold-MSI: Adaptation and validation of an inventory for the measurement of musical sophistication in a Taiwanese sample. Musicae Scientiae. https://doi.org/10.1177/1029864919871987

Loughridge, D. (2021). "Always already technological": New views of music and the human in musicology and the cognitive sciences. Music Research Annual, 2, 1-22.

Margulis, E. H., Wong, P. C. M., Simchy-Gross, R., \& McAuley, J. D. (2019). What the music said: Narrative listening across cultures. Palgrave Communications, 5(1), 1-8. https://doi.org/10.1057/s41599-019-0363-1

McAuley, J.D., Wong, P.C.M., Mamidipaka, A., Phillips, N. \& Margulis, E.H. (In press). Do you hear what I hear? Perceived narrative constitutes a semantic dimension for music. Cognition.

Mehr, S. A., Singh, M., Knox, D., Ketter, D. M., Pickens-Jones, D., Atwood, S., Lucas, C., Jacoby, N., Egner, A. A., Hopkins, E. J., Howard, R. M., Hartshorne, J. K., Jennings, M. V., Simson, J., Bainbridge, C. M., Pinker, S., O’Donnell, T. J., Krasnow, M. M., \& Glowacki, L. (2019). Universality and diversity in human song. Science, 366, eaax0868. https://doi.org/10.1126/science.aax0868

Mignolo, W. D. (2011). The darker side of Western modernity: Global futures, decolonial options. Duke University Press.

Miguel, A. F. (2018). O que é o Musicultura? Um estudo de caso sobre um grupo de pesquisa participativa na Maré, Rio de Janeiro. Rio de Janeiro, 31(2), 143-167.

Moshontz, H., Campbell, L., Ebersole, C. R., IJzerman, H., Urry, H. L., Forscher, P. S., . . Chartier, C. R. (2018). The Psychological Science Accelerator: Advancing psychology through a distributed collaborative network. Advances in Methods and Practices in Psychological Science, 1(4), 501-515. https://doi.org/10.1177/2515245918797607

Müllensiefen, D., Gingras, B., Musil, J., \& Stewart, L. (2014). The musicality of non-musicians: An index for assessing musical sophistication in the general population. PLoS ONE, 9(2). https://doi.org/10.1371/journal.pone.0089642

Muru-Lanning, M. (2020). Vision Mātauranga, eclectic anthropology and the fading empire. In L. George, J. Taori, \& L. T. A. o T. MacDonald (Eds.), Indigenous research ethics: Claiming research sovereignty beyond deficit and the colonial legacy (pp. 53-65). Emerald. https://doi.org/10.1108/s2398-601820200000006004 
Muthukrishna, M., Bell, A. V., Henrich, J., Curtin, C. M., Gedranovich, A., McInerney, J., \& Thue, B. (2020). Beyond Western, Educated, Industrial, Rich, and Democratic (WEIRD) Psychology: Measuring and mapping scales of cultural and psychological distance. Psychological Science, 31(6), 678-701. https://doi.org/10.1177/0956797620916782

Nature Editors. (2020). Achieving diversity in research. Nature. https://www.nature.com/collections/qsgnpdtgbr

Neely, A. (2020). What is your musical IQ? https://www.youtube.com/watch?v=b15BrgretPs

Parker, M. and Kingori, P., 2016. Good and bad research collaborations: Researchers' views on science and ethics in global health research. PloS ONE, 11(10), e0163579. https://doi.org/10.1371/journal.pone.0163579

Powell, M. (2021, February 14). Obscure musicology journal sparks battles over race and free speech. New York Times. https://www.nytimes.com/2021/02/14/arts/musicology-journal-race-freespeech.html

Purzycki, B. G., Henrich, J., Apicella, C., Atkinson, Q. D., Baimel, A., Cohen, E., McNamara, R. A., Willard, A. K., Xygalatas, D., \& Norenzayan, A. (2017). The evolution of religion and morality: a synthesis of ethnographic and experimental evidence from eight societies. Religion, Brain \& Behavior, O(0), 1-32. https://doi.org/10.1080/2153599X.2016.1267027

Rasmussen, A., \& Cowdery, J. (2018). Forum: The lure of universals. SEM Newsletter, 52(2), 7-8. https://cdn.ymaws.com/www.ethnomusicology.org/resource/resmgr/newsletters/SEMNL522.pdf

Russonello, G. (2017, July 11). The unfinished work of Alan Lomax's Global Jukebox. New York Times. $\quad$ https://www.nytimes.com/2017/07/11/arts/music/alan-lomax-global-jukebox-digitalarchive.html

Rzeszutek, T., Savage, P. E., \& Brown, S. (2012). The structure of cross-cultural musical diversity. Proceedings of the Royal Society B: Biological Sciences, 279(1733), 1606-1612. https://doi.org/10.1098/rspb.2011.1750

de Sousa Santos, B. (2007) Beyond abyssal thinking: From global lines to ecologies of knowledge. Review 30 (1): 45 - 89.

Sardo, S. (2017) Shared research practices on and about music: Toward decolonising colonial ethnomusicology, In J. Martí \& S.R. Gútiez Making Music, Making Society (pp. 217 - 238). Cambridge: Cambridge Scholars.

Savage, P. E. (2018). Alan Lomax's Cantometrics Project: A comprehensive review. Music \& Science, 1, 1-19. https://doi.org/10.1177/2059204318786084 
Savage, P. E. (2019). Universals. In J. L. Sturman (Ed.), SAGE International Encyclopedia of Music and Culture (pp. 2282-2285). SAGE Publications. https://doi.org/10.4135/9781483317731.n759

Savage, P. E. (In press). An overview of cross-cultural music corpus studies. In D. Shanahan, A. Burgoyne, \& I. Quinn (Eds.), Oxford Handbook of Music and Corpus Studies. Oxford University Press. https://doi.org/10.31235/osf.io/nxtbg

Schippers, H. \& Grant, C. (eds). (2016). Sustainable futures for music cultures: An ecological approach. New York: Oxford University Press.

Schroeder, D., Cook, J., Hirsch, F., Fenet, S., \& Muthuswamy, V. (Eds.). (2018). Ethics dumping: Case studies from North-South research collaborations. Springer Open.

Sinha, D. (2002). Culture and psychology: Perspective of cross-cultural psychology. Psychology \& Developing Societies, 14(1), 11-25. https://doi.org/10.1177/097133360201400102

Taras, V., Rowney, J., \& Steel, P.. (2009). Half a century of measuring culture: Review of approaches, challenges, and limitations based on the analysis of 121 instruments for quantifying culture." Journal of International Management 15(4), 357-373.

Thompson, W. F., Sun, Y., \& Fritz, T. (2019). Music across cultures. In P. J. Rentfrow \& D. J. Levitin (Eds.), Foundations in music psychology: Theory and research (pp. 503-541). MIT Press.

Thompson et al. (In prep.)

Tran, M., Cabral, L., Patel, R., \& Cusack, R. (2017). Online recruitment and testing of infants with Mechanical Turk. Journal of Experimental Child Psychology, 156, 168-178. https://doi.org/10.1016/j.jecp.2016.12.003

Urassa, M., Lawson, D. W., Wamoyi, J., Gurmu, E., Gibson, M. A., Madhivanan, P., \& Placek, C. (2021). Cross-cultural research must prioritize equitable collaboration. Nature Human Behaviour. https://doi.org/10.1038/s41562-021-01076-X

Vogt, A., Hauber, R., Kuhlen, A. K., \& Abdel Rahman, R. (2021). Internet based language production research with overt articulation: Proof of concept, challenges, and practical advice. PsyArXiv Preprint. https://doi.org/10.31234/osf.io/cyvwf

Woo, M. (2019, November 21). Music may really be a universal language. Inside Science. https://www.insidescience.org/news/music-may-really-be-universal-language

Wood, A. L. C., Kirby, K. R., Ember, C. R., Silbert, S., Daikoku, H., McBride, J., Passmore, S., Paulay, F., Flory, M., Szinger, J., D’Arcangelo, G., Guarino, M., Atayeva, M., Rifkin, J., Baron, V., El Hajli, M., Szinger, M., \& Savage, P. E. (2021). The Global Jukebox: A public database of performing arts and culture. PsyArXiv preprint. https://doi.org/10.31234/osf.io/4z97j 
van Noorden, R. (2020). The ethical questions that haunt facial-recognition research. Nature, 587(7834), 354-358. https://doi.org/10.1038/d41586-020-03187-3

Yong, E. (2018, January 25). A study suggests that people can hear universal traits in music: But some music scholars have doubts. The Atlantic. https://www.theatlantic.com/science/archive/2018/01/the-search-for-universal-qualities-in$\underline{\text { music-heats-up/551447/ }}$ 